\title{
Uncovering co-expression gene network modules regulating fruit acidity in diverse apples
}

\author{
Yang Bai ${ }^{1}$, Laura Dougherty ${ }^{1}$, Lailiang Cheng $^{2}$, Gan-Yuan Zhong ${ }^{3}$ and Kenong Xu ${ }^{1 *}$
}

\begin{abstract}
Background: Acidity is a major contributor to fruit quality. Several organic acids are present in apple fruit, but malic acid is predominant and determines fruit acidity. The trait is largely controlled by the Malic acid (Ma) locus, underpinning which Mal that putatively encodes a vacuolar aluminum-activated malate transporter1 (ALMT1)-like protein is a strong candidate gene. We hypothesize that fruit acidity is governed by a gene network in which Ma1 is key member. The goal of this study is to identify the gene network and the potential mechanisms through which the network operates.
\end{abstract}

Results: Guided by Ma1, we analyzed the transcriptomes of mature fruit of contrasting acidity from six apple accessions of genotype Ma_ (MaMa or Mama) and four of mama using RNA-seq and identified 1301 fruit acidity associated genes, among which 18 were most significant acidity genes (MSAGs). Network inferring using weighted gene co-expression network analysis (WGCNA) revealed five co-expression gene network modules of significant $(P<0.001)$ correlation with malate. Of these, the Ma1 containing module (Turquoise) of 336 genes showed the highest correlation (0.79). We also identified 12 intramodular hub genes from each of the five modules and 18 enriched gene ontology (GO) terms and MapMan sub-bines, including two GO terms (GO:0015979 and GO:0009765) and two MapMap sub-bins (1.3.4 and 1.1.1.1) related to photosynthesis in module Turquoise. Using Lemon-Tree algorithms, we identified 12 regulator genes of probabilistic scores 35.5-81.0, including MDP0000525602 (a LLR receptor kinase), MDP0000319170 (an IQD2-like CaM binding protein) and MDP0000190273 (an EIN3-like transcription factor) of greater interest for being one of the 18 MSAGs or one of the 12 intramodular hub genes in Turquoise, and/or a regulator to the cluster containing Ma1.

Conclusions: The most relevant finding of this study is the identification of the MSAGs, intramodular hub genes, enriched photosynthesis related processes, and regulator genes in a WGCNA module Turquoise that not only encompasses Ma1 but also shows the highest modular correlation with acidity. Overall, this study provides important insight into the Ma1-mediated gene network controlling acidity in mature apple fruit of diverse genetic background.

Keywords: Malus, Fruit acidity, Ma1, Aluminum-activated malate transporter1 (ALMT1), Gene network

\section{Background}

Apple fruit acidity refers to the sensory intensity of tartness of fruit flesh tissues. The stronger the tartness taste, the higher the fruit acidity levels. Chemically, fruit acidity can be quantified by measuring fruit juice $\mathrm{pH}$ and/or titratable acidity (TA). It has been shown that several organic acids are present in mature apple fruit, including malic acid, quinic acid, citric acid and others, but malic acid consists of more than $90 \%$ of the total [1-3], thereby largely determining fruit acidity. For dessert apples, fruit acidity could be

\footnotetext{
* Correspondence: kx27@cornell.edu

${ }^{1}$ Horticulture Section, School of Integrative Plant Science, Cornell University, New York State Agricultural Experiment Station, Geneva, NY 14456, USA Full list of author information is available at the end of the article
}

grouped into three categories by TA: low $(<3.0 \mathrm{mg} / \mathrm{ml})$, normal $(3.0-10.0 \mathrm{mg} / \mathrm{ml})$, and high $(>10.0 \mathrm{mg} / \mathrm{ml})$, and categories high and low are not acceptable [4]. This makes fruit acidity an essential quality component not only for determining the fate of existing varieties, but also for prompting routine evaluations of acidity levels in apple breeding as genotypes of fruit acidity beyond the acceptable normal range can make up $25-50 \%$ in breeding populations. As a result, fruit acidity has long been an important subject area of investigations in apple genetics.

Apple fruit acidity is primarily controlled by the major gene or QTL on chromosome 16, called Malic acid $(M a)$ alongside a significant QTL on chromosome 8 and a few other QTLs with relatively smaller effects [4-9]. With 
regard to the allelic interactions at the major QTL $M a$, normal/high acid allele $(M a)$ is largely dominant over the low acid allele (ma). Genotypes MaMa and Mama have fruit of normal to high acidity while genotype mama of low acidity with little or none commercial value. To focus on the most important genetic factor, we [10] and others [11] have recently isolated the major QTL $M a$. The common findings were that the $M a$ locus harbors two new members of the Aluminum-activated Malate Transporter1 (ALMT1) gene family, called $M a 1$ and $M a 2$. The studies further found that $M a 1$ was expressed in significantly positive correlation with fruit acidity levels while the expression of $M a 2$ was barely detectable in both high and low acid fruit, suggesting that it was gene $M a 1$ rather than $M a 2$ that was the very gene underlying $M a[10,11]$. In addtion, a detailed analysis of the allele specific DNA sequences of Ma1 indicated that a single base mutation that would stop the protein translation process prematurely was almost completely associated with low acidity in a diverse set of apple germplasm studied, suggesting that the low acidity is caused by the malfunction of the MA1 protein due to the deduced truncation at the C-terminus [10].

These latest findings have markedly increased our understanding on fruit acidity, but many questions remain to be answered. For example, how does the landscape of transcriptomes differ between genotypes $\mathrm{Ma} \mathrm{a}_{-}$(MaMa and Mama) and mama at maturity stage? If fruit acidity is governed by a gene network in which $M a 1$ is the genetic determinant, what would the other possible members in the network? What are the potential biological process and/or regulatory mechanisms responsible for the contrast acidity levels between genotype groups $M a_{-}$and mama? The development of mRNA sequencing (RNAseq) technology that unlocks the power of high throughput of next generation sequencing has provided an ideal means to address these questions. Since its inception $[12,13]$, RNA-seq has been rapidly adapted in transcriptomics studies in plants such as Arabidopsis [14], grape [15], maize [16] and rice [17]. In apple, RNAseq based studies have recently been reported as well [18-24]. More importantly, to begin resolving the low coverage issue of the current version of apple reference transcriptome, we have improved it with RNAseq reads from fruit of Golden Delicious (GD), the source of the reference genome [25], which is available at the Genome Database for Rosaceae (GDR) [26]. We have also used the improved reference transcriptome through RNA-seq approach to construct a co-expression gene network associated with developmental regulation of malate levels varying from 5.2 to $14.5 \mathrm{mg} / \mathrm{g}$ fw (normal to high acidity) in developing fruit of 'Golden Delicious' of genotype Mama [24].

The objectives of this study are to address the questions aforementioned. To do so, we first sequenced 30
RNA-seq libraries representing transcriptomes of mature fruit from ten apple varieties of genotypes $M a M a$, and Mama and mama, and then mapped the RNA-seq reads against the improved apple reference transcriptome [25]. Using $M a 1$ as a guide gene, a series of downstream analyses was conducted, leading to identification of weighted co-expression gene network modules significantly correlated with malate, most significant acidity genes, intramodular hub genes, regulator genes, enriched gene ontology (GO) terms and MapMan subbins, and others. To the best of our knowledge, this is first report attempting to understand the Ma1-mediated gene network regulating fruit acidity in apples of diverse genetic background.

\section{Methods}

\section{Plant materials and fruit acidity quantification}

Ten apple varieties of known genotype at the $M a$ locus were chosen, including four of mama-Britegold (B), Sweet Delicious (S), Novosibirski Sweet (N) and PI323617 (P), four of Mama-Fuji (F, Red Sport Type 2), Rome Beauty Law (R), Cox's Orange Pippin (C) and Jonathan (J), and two of MaMa-Empire (E) and Granny Smith (G). Genotypes Mama and MaMa were jointly designated $\mathrm{Ma} \mathrm{a}_{-}$to represent a group that had at least one functional allele of $M a 1$. The trees were budded onto rootstock P22 and grown in a research orchard of Cornell University at Geneva, New York. Fruit of three replicates per variety and 8-10 fruit per replicate were harvested at maturity from 2 to 3 trees in fall 2012 (Additional file 1: Table S1). Each fruit was cross-sectioned into two halves: one half were used for maturity evaluation and fruit juice extraction, and the other half were sliced and immediately frozen in liquid nitrogen for RNA isolation and for quantitation of malate and other metabolites. The evaluation of fruit maturity and fruit acidity was conducted as previously described [4]. Fruit of Cornell Starch Index 4.0-6.0 [27] were considered matured and only matured fruit (with the core removed) were used for analysis. Two indicators of fruit acidity were measured: $\mathrm{pH}$ by a $\mathrm{pH}$ meter (Accumet AB15, Fisher Scientific, PA, USA) and titratable acidity (TA, malic acid equivalent) by an autotitrator (Metrohm 848 Titrino Plus with 869 compact sample changer, Metrohm, Herisau, Switzerland).

Fruit organic acids were extracted and measured using an Agilent 7890A GC/5795C MS (Agilent Technology, Palo Alto, CA, USA) with the same configurations and settings as described previously [24]. Metabolites were identified by comparing fragmentation patterns with those in mass spectral libraries and quantified based on standard curves for each metabolite and the internal standard ribitol. 


\section{RNA-seq library construction and data analysis}

Fruit RNA isolation and RNA-seq library construction were performed as previously described [25]. Briefly, total RNA was isolated from $3 \mathrm{~g}$ of ground fruit flesh tissues and then treated with DNase I (amplification grade, Invitrogen/Life Technologies, Carlsbad, CA). For mRNA isolation and strand specific RNA-seq library construction, NEBNext Poly(A) mRNA Magnetic Isolation Module and NEBNext Ultra Directional RNA Library Prep Kit for Illumina (New England Biolabs, Ipswich, MA) were used. The mRNA was isolated from $5 \mu \mathrm{g}$ of total RNA and was fragmented at $94{ }^{\circ} \mathrm{C}$ for $10 \mathrm{~min}$. The first strand cDNA was reverse transcribed from the fragmented mRNA with dATP mix and second strand cDNA was synthesized from the first strand cDNA with dUTP mix. The resulting double strand cDNA was end-repaired, adaptor-ligated and size-selected, and then followed by the USER enzyme digestion of the second strand cDNA. The intact modified first strand cDNA was PCR-enriched for 14-16 cycles to obtain the individual sequencing ready RNA-seq libraries. These libraries were multiplexed in equal amount for single end 100-base sequencing in three lanes of HiSeq 2000 (Illumina, San Diego, CA) at the Cornell University Biotechnology Resource Center (Ithaca, NY). The ten apple varieties were sequenced in three biological replicates with one replicate per lane.

Illumina sequencing of the pooled RNA-seq libraries generated 30 FASTQ files of sequences with a total of 615.6 million raw reads (Additional file 2: Table S2). The raw reads were fed into Bowtie [28] to remove bar codes and adapters and then aligned to the rRNA reference sequences allowing up to three mismatches, which were downloaded from the SILVA rRNA database (http:// www.arb-silva.de) [29]. The rRNA depleted reads were imported into CLC Genomics Workbench v6.5 (CLCBio, Cambridge, Massachusetts) to trim low quality reads and/ or bases using the quality limit of 0.05 and the ambiguous limit of 1 . The resultant clean and high quality reads were mapped by CLC Genomics Workbench (using the minimum similarity fraction of 0.98 , the minimum length fraction of 0.8 and the maximum number of hits of 10) against the improved apple reference transcriptome [25], which is available at URL http://www.rosaceae.org/species/malus/malus_x_domestica/CU_RNA_ seq_genes of GDR [26]. In addition to 53,654 of the 63,541 originally predicted genes or MDPs (the three letters prefixing gene IDs in apple), the reference transcriptome includes 17,524 novel transcripts [25]. For convenience, these novel transcripts will be referred to 'genes' and named 'G\#\#\#\#\#s' as in 'G101234', and 'MDP0000' (e.g. MDP0000252114) in the original gene IDs will be abbreviated to ' $M$ ' (e.g. M252114) hereafter. Gene expression levels were calculated and represented by reads per kilobase of exon model per million mapped reads (RPKM) [12]. Genes of RPKM $>0.3$ were considered expressed according to a previous study [30].

\section{Identification of fruit acidity associated genes (FAAGs)}

Fruit acidity associated genes (FAAGs) were defined for those that were differentially expressed between genotype groups $\mathrm{Ma}$ and mama and/or those that were expressed similarly as the guide gene Ma1. To identify differentially expressed genes (DEGs) between the two genotype groups, the gene expression data RPKMs were subjected to Baggerly's test [31]. To control the false discovery rate (FDR), the original P values in Baggerly's test were adjusted for multiple testing using Benjamini-Hochberg correction [32]. The cutoff for declaring a gene expressed differentially was $\mathrm{P}_{\mathrm{FDR}}<0.05$. However, any genes with an RPKM range $<5$ in the 29 RNA seq samples were removed from the list. To identify genes expressed similarly as Ma1, two filters were applied: 1) the mean difference in RPKM between the two genotype groups was greater than ten (8.53 for Ma1), and 2) the fold change in mean RPKM between the two genotype groups was greater than 1.5 (1.69 for Ma1). Genes passed both filters were considered of similar expression as Ma1.

\section{Inferring fruit acidity associated co-expression gene network modules}

The co-expression gene network modules (highly coexpressed clusters of genes) were inferred from the fruit acidity associated genes (FAAGs) using weighted gene co-expression network analysis (WGCNA), an R package [33]. Before network inferring, the expression data (RPKM) were normalized by square root transformation. The automatic one-step network construction and module detection method with default settings were used, which include an unsigned type of topological overlap matrix (TOM), a power $\beta$ of 6 , a minimal module size of 30 , and a branch merge cut height of 0.25 . The module eigengene (the first principal component of a given module) value was calculated and used to test the association of modules with acidity in the ten genotypes of 29 samples. Gene significance (GS, the correlation between gene expression and acidity), total network connectivity (kTotal), and module membership (MM) that is also known as eigengenebased intramodular connectivity $(\mathrm{kME})$, were calculated for each of the 1301 FAAGs in the ten modules. The most significant module (Turquoise) was visualized using Cytoscape 3.1 [34] and were also analyzed using Network Analyzer [35], a Cytoscape plugin.

\section{Identification of regulator genes}

Detection of regulator genes was performed using LemonTree software [36], which is an improved and 'one-stop shop' version of the stochastic Bayesian module network algorithm Learning Module Networks (LeMoNe), which 
requires run scripts on both MATLAB and Perl [37]. Using expression data from the 1301 FAAGs, ten independent Gibbs sampler runs [38] were conducted with Lemon-Tree. From these ten runs, we were able to generate a single set of tight independent clusters, and then select 50 (Clusters $0-49$ ) of at least ten genes per cluster for testing 96 candidate regulators. The candidate regulators were genes annotated as transcription factors or signal transducers in GO terms and/or MapMan (sub-) bins in the 1301 genes. We considered candidate regulators as regulators when their probabilistic $(P$.) scores associated with their assignment to any of the 50 tight clusters were within top $1 \%$.

\section{Functional annotation and enrichment analysis of WGCNA modules}

We previously used both MapMan bins [39] and Gene Ontology (GO) terms to annotate the improved apple reference transcriptome [24, 25]. Briefly, for annotation with MapMan (sub-) bins, several databases were BLASTsearched with the apple reference transcriptome of 71,178 genes using Mercator, a web-based search tool [40]. The searched databases include: TAIR-Arabidopsis TAIR proteins (release 10), PPAP-SwissProt/UniProt Plant Proteins, CHLAMY-JGI Chlamy release 4 Augustus models, ORYZA-TIGR5 rice proteins, KOG-Clusters of orthologous eucaryotic genes database, CDD- conserved domain database, and IPR-interpro scan. For GO term annotations, relevant information for apple genes (MDPs) is available at GDR [26] and was directly adapted. However, GO terms for the new transcripts [25] were obtained by BLAST2GO program [41] together with BLASTx search against the NCBI Reference Proteins database (cutoff $E=10^{-6}$ ). Enrichment analyses of the WGCNA modules were all conducted by hypergeometric tests using CLC GW against the same background of 39,679 expressed genes. The hypergeometric test is similar to the unconditional GOstats test [42], and the cutoff for significant enrichment is $\mathrm{P}_{\mathrm{FDR}}<0.05$. Depending upon actual test, the background was defined by 39,679 annotated genes in MapMan (sub-) bins, by 16,153 in GO biological process (BP), or by 21,612 in GO molecular functions (MF).

\section{qRT-PCR analysis}

qRT-PCR analyses were conducted with the same total RNA samples (after DNase I treatment) used for the RNAseq library construction. Two micrograms of total RNA were reverse transcribed using the Superscript III RT module (Invitrogen/Life technology, Carlsbad, CA). The resulting first strand cDNA was diluted by 5 -fold and used as templates for qRT-PCR analysis on a LightCycler 480 (Roche, Indianapolis, IN), where an apple actin gene (EB136338) was served as reference. The primer sequences for the reference gene and the eight target genes were listed (Additional file 3: Table S3). The actual qRT-PCR reactions and subsequent expression analysis were conducted similarly as described previously [24].

\section{Statistical analyses}

Statistical analyses, such as ANOVA, Student's $t$ test and Tukey's HSD (honest significant difference) test, were performed using JMP Pro10 (SAS, Cary, NC).

\section{Results}

Fruit metabolite profiling and acidity evaluation

Fruit metabolite profiling was conducted using GC-MS with three biological replicates in the ten apple varieties. A total of 19 metabolites were quantified, including 12 soluble sugars and seven organic acids. Among the 12 sugars, only sorbitol showed a significantly $(P=0.026$, ANOVA) higher concentration in genotype group mama $(7.4 \pm 2.0 \mathrm{mg} / \mathrm{g} \mathrm{FW})$ than in $\mathrm{Ma}$ ( $(5.4 \pm 1.9 \mathrm{mg} / \mathrm{g} \mathrm{FW})$. Other sugars, especially the highly abundant fructose, sucrose and glucose, had similar levels across these varieties. Among the seven organic acids (malate, dehydroascorbate, maleate, succinate, fumarate, citrate and quinate), malate was the most abundant $(87.2 \pm 7.2 \%$ of the total acidity) while quinate $(8.3 \pm 6.7 \%)$ and maleate $(2.5 \pm 0.8 \%)$ were distantly followed as the second and third abundant, respectively. Malate concentrations varied significantly $(P=1.03 \mathrm{E}-9)$ in the ten apple varieties (Fig. 1a), and were more than 3-fold higher in genotype

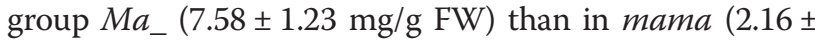
$0.41 \mathrm{mg} / \mathrm{g} \mathrm{FW}$ ) (Fig. 1b). The concentrations of maleate, succinate, fumarate and citrate were also significantly higher in genotype group $M a_{-}$than in mama $(P<0.0001)$, but they were at much lower levels (at $\mu \mathrm{g} / \mathrm{g}$ FW) (Fig. 1c). However, quinate showed no significant difference between groups $\mathrm{Ma}$ and mama. The trend of fruit juice titratable acidity (TA) was similar to that of malate in the ten varieties (Fig. 1a, b) while an expected reverse trend was observed for fruit juice $\mathrm{pH}$ (Fig. 1d).

\section{Transcriptome analysis in genotype groups $\mathrm{Ma}$ and mama}

RNA-seq data analysis was performed using the improved apple reference transcriptome [25]. After removing reads derived from rRNA and/or of low quality, the reads for RNA-seq mapping were 462.9 million in total (Additional file 2: Table S2). Overall the total mapped and uniquely mapped reads were 331.6 million (70.5\%) and 281.4 million (59.8\%), respectively. The mean mapped reads per sample were $11.1 \pm 4.1$ million in total and $9.4 \pm 3.5$ million in unique. However, the sample for replicate I of Granny Smith was excluded from the downstream analysis as $75.0 \%$ of its raw reads was 
(A)

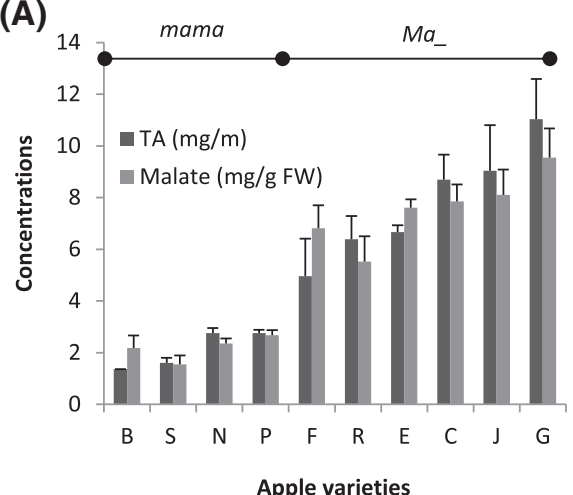

(D)

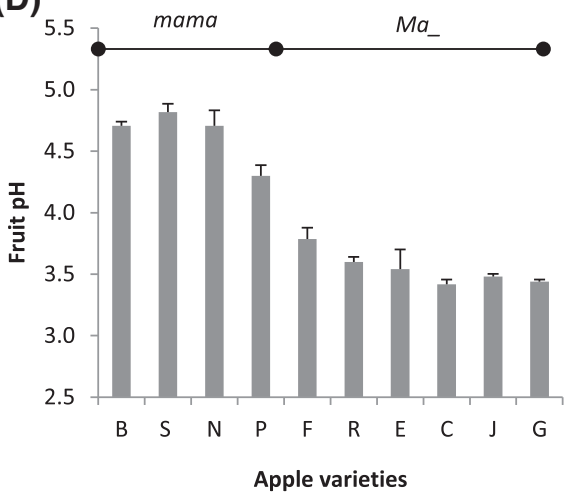

(B)

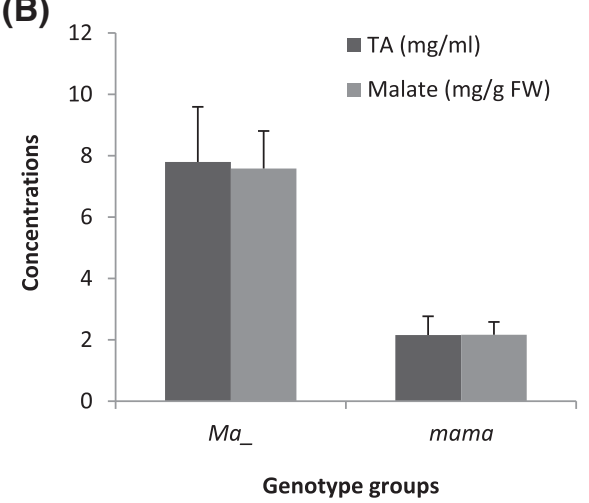

(C)

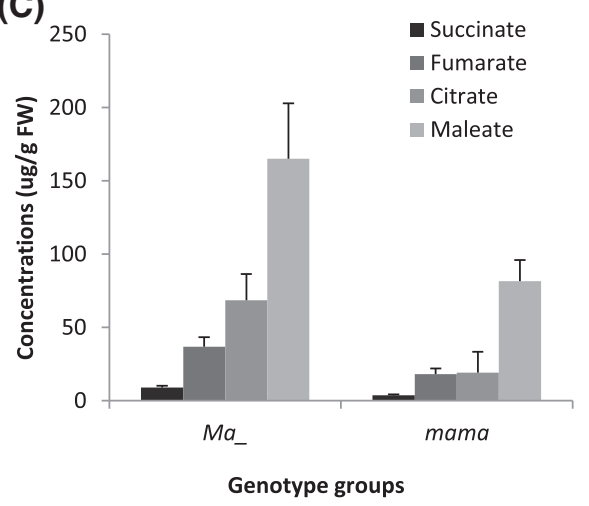

Fig. 1 Evaluation of fruit acidity and causal organic acids. Standard deviations were shown with the error bars. a TA and malate concentrations measured in the ten apple varieties, which were abbreviated as the following: B Britegold, N Novosibirski Sweet, P PI323617, S Sweet Delicious, C Cox's Orange Pippin, E Empire, F Fuji, G Granny Smith, J Jonathan, R Rome Beauty Law. The first four are of genotype mama whereas the last six of genotype $\mathrm{Ma}_{-}$. b Mean TA and malate concentrations in genotype groups $\mathrm{Ma}$ _ and mama. c Concentrations of succinate, fumarate, citrate and maleate in genotype groups $\mathrm{Ma}_{-}$and mama. $\mathbf{d}$ Fruit $\mathrm{pH}$ readings in the ten apples

removed by the rRNA and quality filters (Additional file 2: Table S2).

The maximum number of expressed genes was 52,102 in this study, a tally counting all genes of RPKM $>0.3$ in at least one of the 29 samples. However, measuring by the threshold in group means (RPKM $>0.3$ in at least one of the two group means), the expressed genes were 39,679 (Fig. 2a). Of these, 35,618 were expressed in both genotype groups, and 1866 and 2195 were specifically in $M a_{-}$and mama, respectively (Fig. 2a).

The total mapped reads of the expressed genes were 11.5 \pm 3.9 million per sample in the $M a_{\text {_ group }}$ and $11.1 \pm 3.3$ million per sample in mama (Fig. 2b). The uniquely mapped reads per sample in the $M a_{-}$and mama groups were $9.8 \pm 3.3$ million and $9.4 \pm 2.8$ million, respectively (Fig. 2b).

\section{Identification of malate associated co-expression gene network modules using WGCNA}

Transcriptome-wide comparison of the 39,679 expressed genes revealed 1301 fruit acidity associated genes (FAAGs), including 633 (including Ma1) of differentially
$\left(\mathrm{P}_{\mathrm{FDR}}<0.05\right)$ expressed genes (DEGs) between groups $M a$ and mama, and 668 expressed similarly as the guide gene Ma1 (Additional file 4: Table S4). Using WGCNA, a co-expression gene network was constructed from the 1301 FAAGs (Fig. 3a). Within the network, the total network connectivity of genes showed a significant correlation $\left(\mathrm{r}^{2}=0.1952, p=4.02 \mathrm{E}-12\right)$ with the gene significance for malate (Fig. 3b), suggesting that more connected genes would have higher gene significance for acidity in the 1301 FAAGs.

Further WGCNA analysis identified ten network modules in the co-expression network, designated Turquoise, Black, Brown, Pink, Magenta, Red, Green, Blue, Yellow, and Grey (for four unassigned genes) (Fig. 3a, c). Investigating the relationships between module eigengene and acidity uncovered that the correlation coefficients varied widely from -0.67 to 0.79 in malate and from -0.55 to 0.75 in TA (Fig. 3c). The eigengenes (Fig. 4a, Additional file 5: Figure S1A) of five modules Turquoise, Black, Brown, Blue and Yellow showed significant correlations $(p<0.001)$ with malate, suggesting these five modules had greater relevance in fruit acidity although Blue and Yellow were negatively 


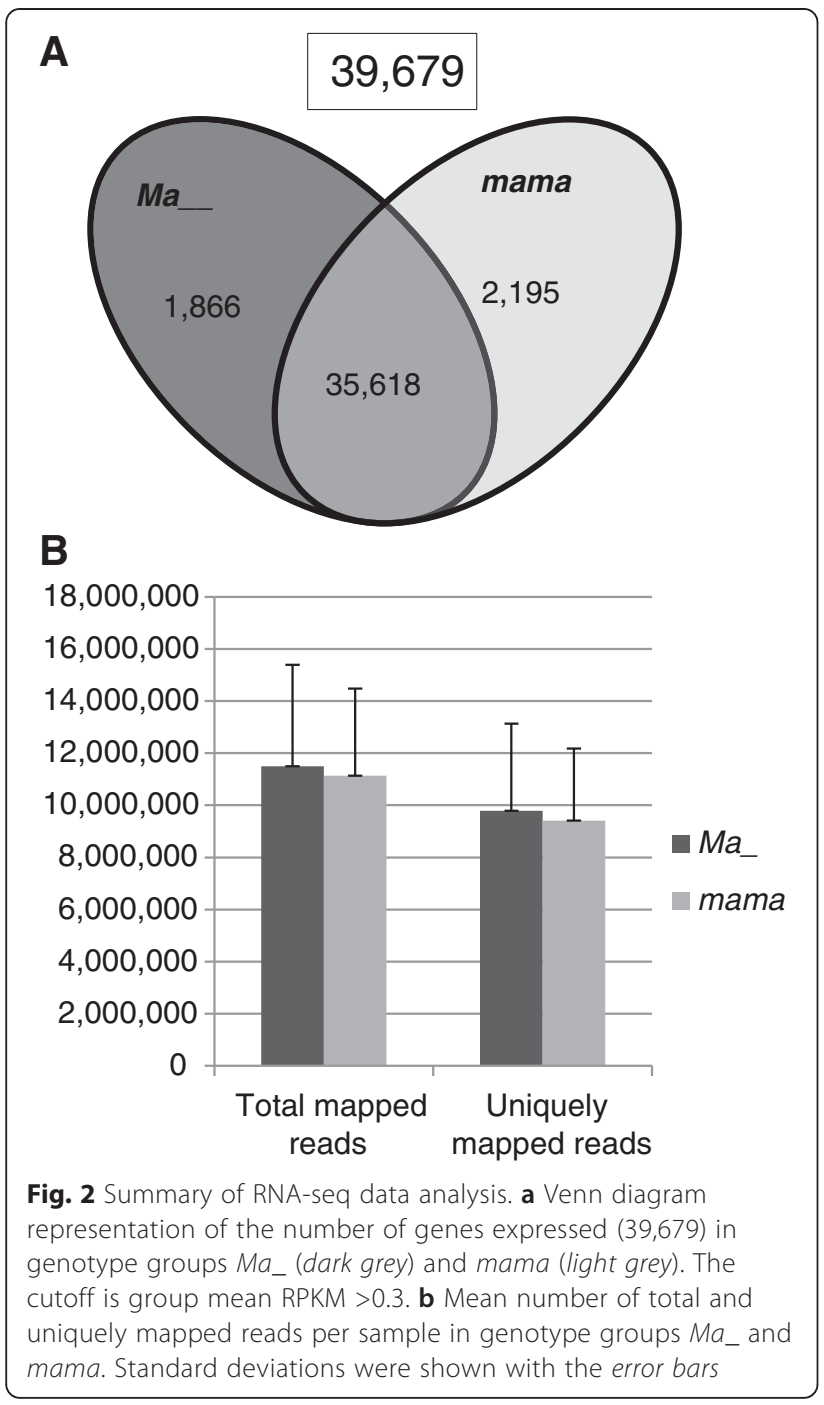

correlated (Fig. 3c). The five modules comprised 943 genes with module Turquoise being the largest of 336 genes. Notably, the guide gene Ma1 was assigned to Turquoise, which also had the highest modular correlation $(r=0.75-0.79)$ with acidity (Fig. 3c). Inspecting the correlation between the module memberships (MM) and gene significance (GS) revealed that all modules were significant except Blue (Fig. 4b, Additional file 5: Figure S1B). Again, module Turquoise had the most significant correlation between MM and GS ( $r=0.49, p=1.1 \mathrm{E}-21)$, indicating genes of higher MM values were more likely of greater GS in Turquoise than in others.

\section{Identification of the most significant acidity genes (MSAGs)}

The 1301 FAAGs had a range of absolute gene significance (GS) for malate from 0.004 to 0.911 (Fig. 3b). We define that the most significant acidity genes (MSAGs) are those of GS for malate $\geq 0.801$, the observed GS of the guide gene Ma1. This allowed to identify 18 MSAGs (Fig. 5a, Additional file 6: Table S5), including three transporters (M282275, M834327 and Ma1), two receptor kinases (M525602, M651862), one Ethylene-Insensitive3 (EIN3)-like transcription factor (M190273), one pyruvatedehydrogenase complex component E2 (M727725), one endo-1,4-beta-xylanase/ hydrolase (M225641), one glutam ine synthetase cytosolic isozyme (G202922), one photosys tem II subunit R (M800352), and seven (M364253, M442 350, M345601, G103681, M230253, G106959, G104167 and G104764) of unknown function. The 18 MSAGs were distributed in three modules with 12 in Turquoise, three in Black and another three in Blue (Fig. 5b, Additional file 6: Table S5), further indicating module Turquoise is of greater relevance in acidity.

\section{Identification of intramodular hub genes and evaluation of their total network connectivity changes in genotype groups $\mathrm{Ma}$ and mama}

To identify intramodular hub genes, we similarly used the guide gene Ma1, which had an MM of 0.894, ranking 11th in module Turquoise. Based on this information, we defined top 12 genes in MM values from each of the five modules as intramodular hub genes, leading to identification of 60 hub genes (Fig. 6, Additional file 7: Table S6). To evaluate how the 60 hub genes behaved in genotype groups $\mathrm{Ma} a_{-}$and in mama, their respective total network connectivity (kTotal) were calculated and compared with the overall group of both genotypes (Fig. 6, Additional file 7: Table S6). Most of the 60 hub genes showed a large increase in kTotal in mama while a marked reduction in Ma_, suggesting a major pattern of negative correlation between the hub genes' kTotal changes and acidity levels (Fig. 6). This pattern was true in modules Blue and Yellow (both negatively correlated with acidity), largely true in Brown and Black (both positively correlated with acidity), but largely untrue in Turquoise (most positively correlated with acidity), seemingly providing another means depicting the module-acidity relationship shown early (Fig. 3c).

There were three additional patterns in kTotal changes (Fig. 6): The first was observed only on M138489 in Turquoise, i.e. a reverse trend by having an increased kTotal in Ma and a decreased kTotal in mama. M138489 encodes a leucine-rich repeat receptor kinase (Fig. 6). The second was represented by Ma1, i.e. the kTotal was decreased in both $M a_{\text {_ }}$ and mama, suggesting reduced kTotal of Ma1 in mama might have important ramifications for low acidity. This pattern was also observed on M319170 (encoding an AtIQD2 like calmodulin ( $\mathrm{CaM}$ ) binding protein involved in calcium signaling), M423596 (a homeobox transcription factor) and M183277 (a hypothetic protein) in Turquoise, M497333, M304911 and G100038 in Black, and M365732 and M257862 in Brown (Fig. 6, Additional file 7: Table S6). The third pattern was characterized by an increased kTotal 


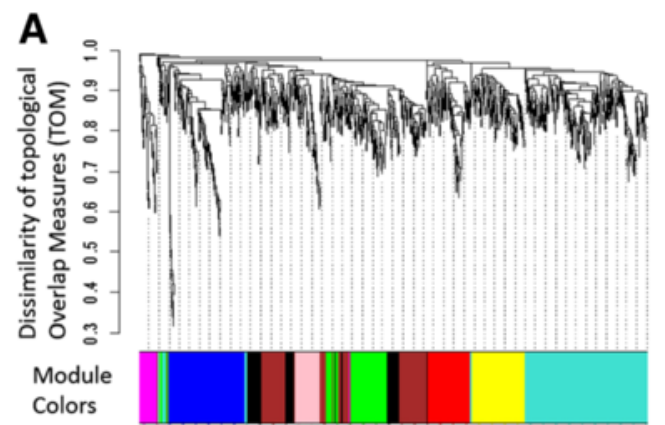

B

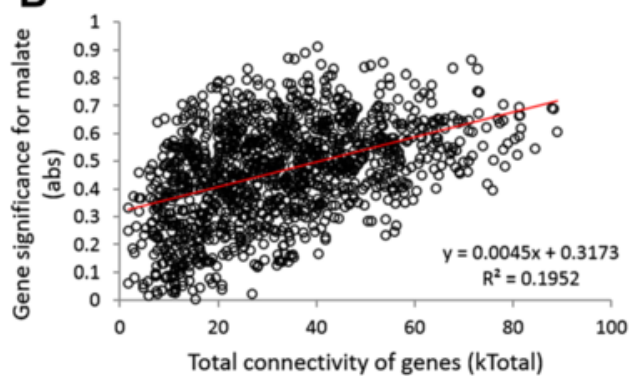

C

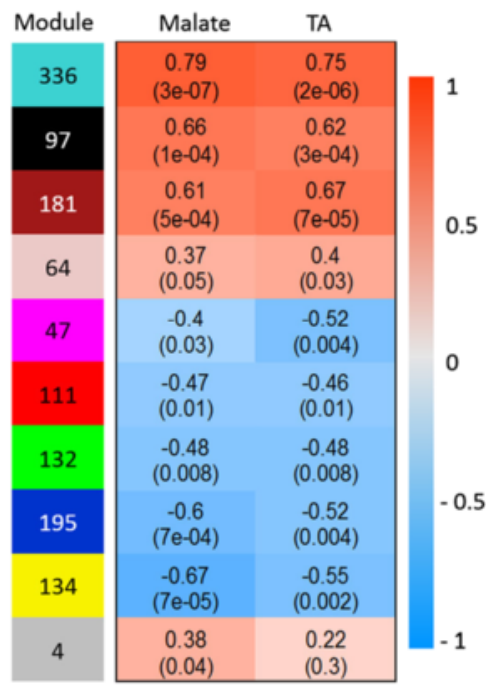

Fig. 3 Weighted gene co-expression network analysis (WGCNA) of fruit acidity associated genes (FAAGs) in the Ma_ and mama genotype groups. a Hierarchical cluster tree showing ten modules of co-expressed genes. Each of the 1301 FAAGs is represented by a leaf in the tree while each of the ten modules by a major tree branch. The lower panel shows modules in designated colors. $\mathbf{b}$ Correlation between gene total network connectivity and absolute gene significance for malate in the whole co-expression gene network. c Module-fruit acidity correlations and corresponding p-values (in parenthesis). The left panel shows ten modules (Turquoise, Black, Brown, Pink, Magnate, Red, Green, Blue, Yellow and Grey) and the number of their member genes. The right panel is a color scale for module trait correlation from -1 to 1

in both Ma_ and mama, which was observed on M189832 (a metalloendopeptidase in protein degradation), M314954 (a WD-40 repeat family protein) and G102909 (a hypothetical protein) in Turquoise, and G302730 and M329552 in Black (Fig. 6, Additional file 7: Table S6).
To graphically view the intramodular connectivity of these hub genes, the 12 hub genes along with other coexpressed (WGCNA edge weight $>0.10$ ) genes in module Turquoise was presented using Cytoscape (Fig. 7a). It showed that the 12 hub genes were of the most edges from

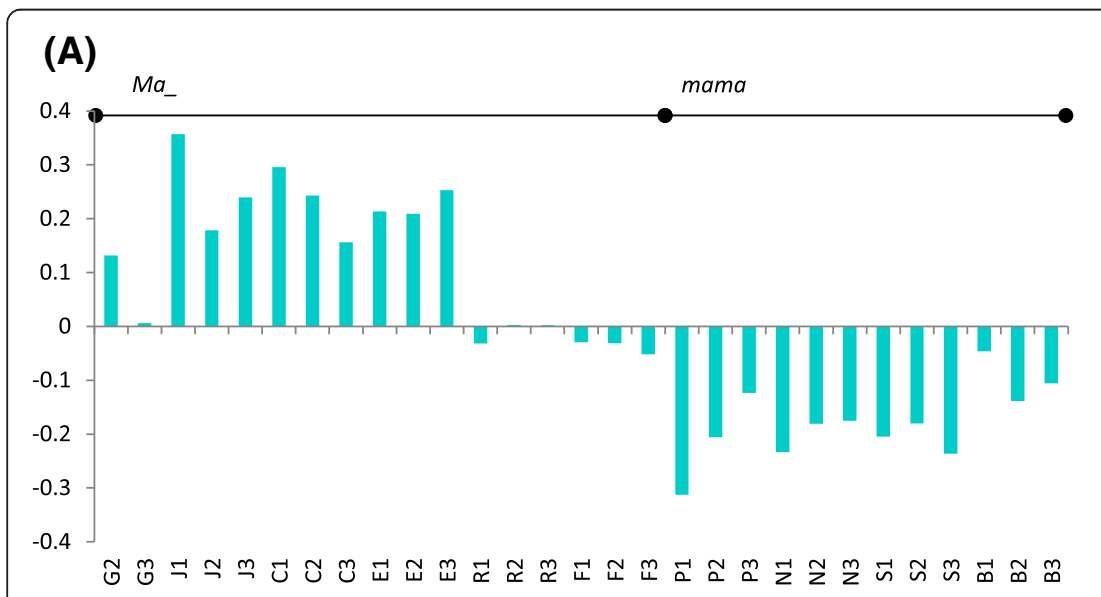

(B)

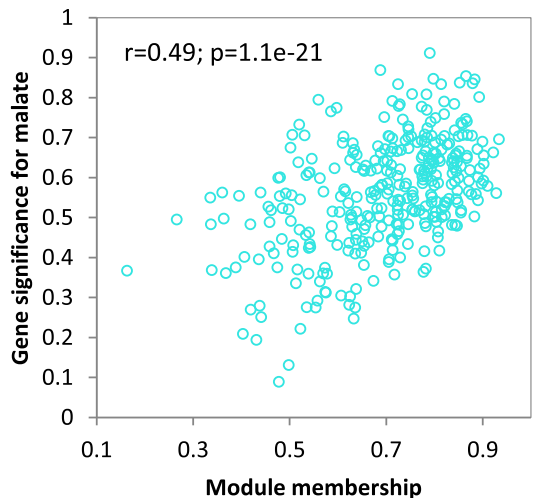

Fig. 4 Analysis of module Turquoise. a Module eigengene values across the 29 samples, including 17 in Ma_on left and 12 in mama on right. Samples are represented by the combination of a letter (abbreviated cultivar name, see Fig. 1's legend) and a number (replicate 1, 2 or 3). b Correlation between module membership (MM) and gene significance (GS) for malate 


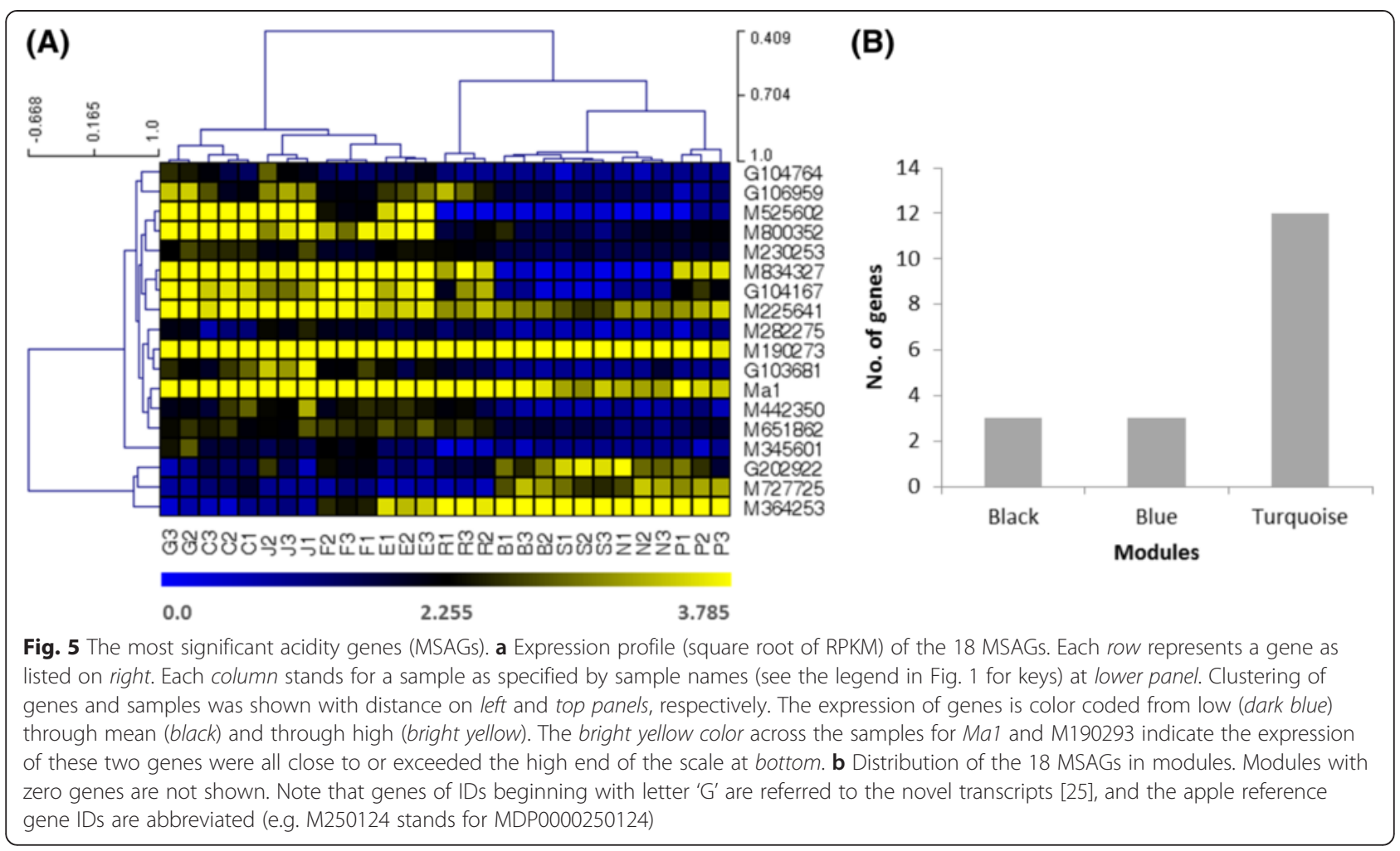

164 to 254 (Fig. 7b) and were in the module network core (Fig. 7a).

\section{Identification of regulator genes}

Regulator genes are not readily identifiable from the WGCNA co-expression network modules as they are nondirected. To identify regulator genes, we used Lemon-Tree software [36] first to generated 50 tight clusters (Clusters 0-49) covering 839 of the 1301 FAAGs through ten Gibbs sampler runs [38], and then to examine the 96 candidate regulators (transcription factors or signal transducers annotated in the 1301 FAAGs) with the clusters. To statistically validate the results obtained from the 96 candidate regulators, another 96 randomly selected genes were used as control (CK) and examined in parallel with the 96 candidate regulators. As expected, the probabilistic $(P$.) scores for genes assigned as a regulator for the 50 tight clusters showed significant difference ( $p=0$ in $\mathrm{z}$ test) between the

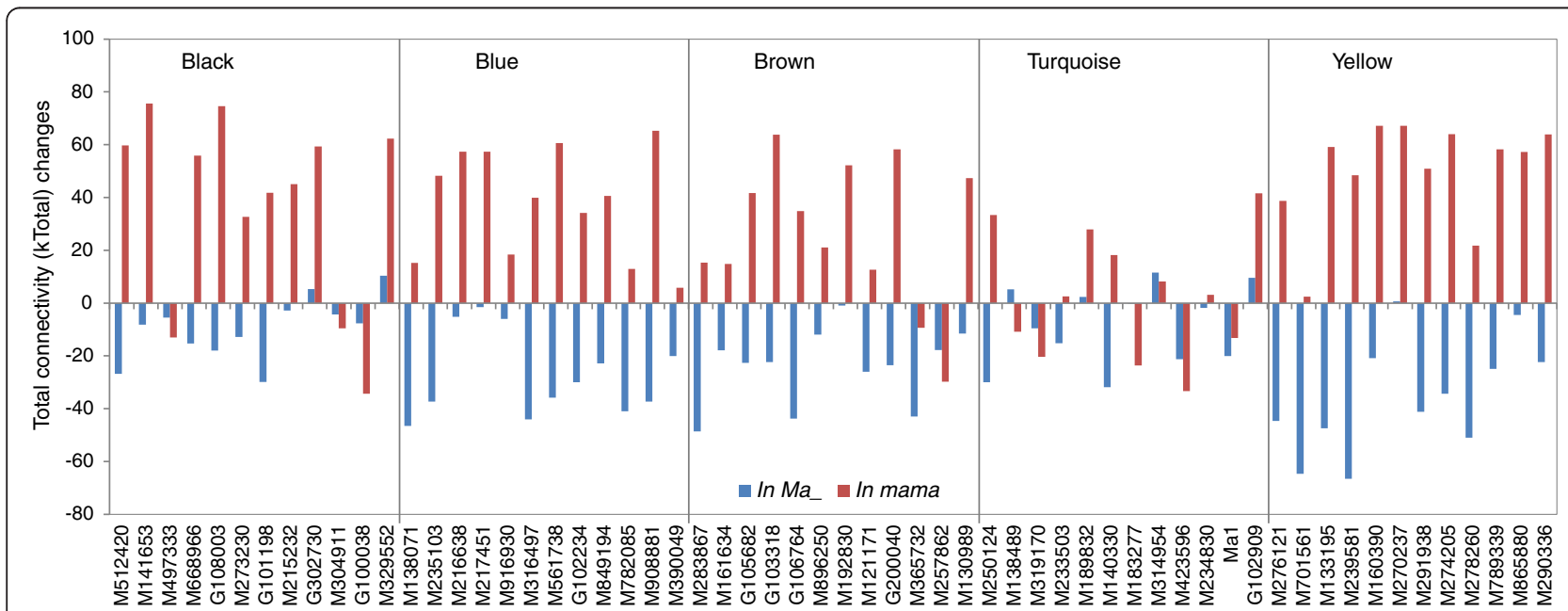

Fig. 6 Intramodular hub genes and their total network connectivity changes in genotype groups Ma_and mama when compared with the combined groups of both genotypes. Shown are modules Black, Blue, Brown, Turquoise and Yellow 


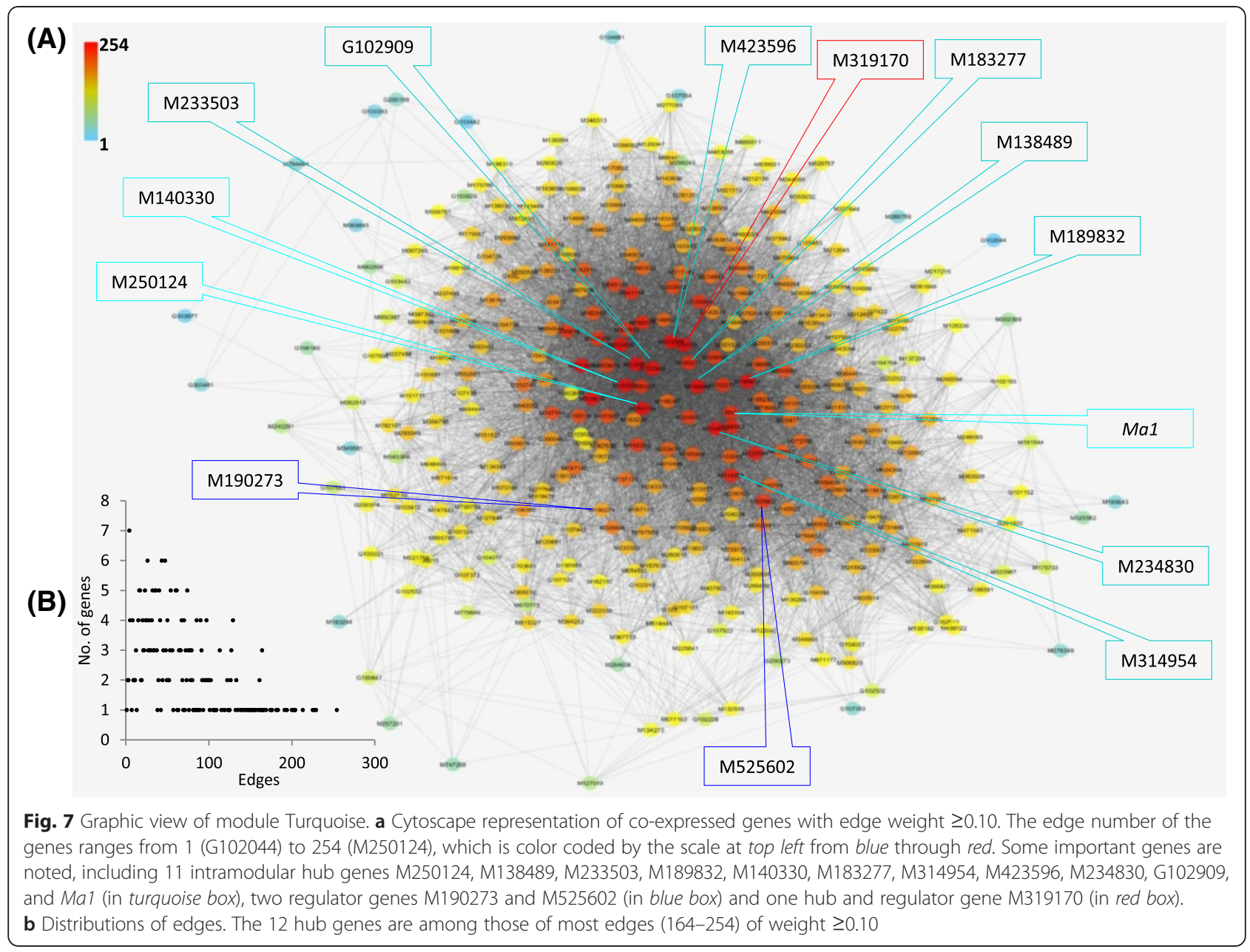

96 candidate regulators (0.1-81.0) and CK (0.1-6.3). At the top $1 \%$ of $P$. scores (35.5-81.0), 12 of the 96 candidate regulators were identified as regulators and assigned to 21 of 50 tight clusters (Additional file 8: Table S7). Of the 12 regulators, five (M190273, M525602, M319170, M239684 and M134341) were from module Turquoise (Fig. 8, Additional file 9: Figure S2, Additional file 10: Figure S3), two (M753318 and M175481) from Brown (Additional file 10: Figure S3), and five from modules Green, Red and Pink which were irrelevant for acidity (Additional file 8: Table S7). Below are brief descriptions of three regulator genes, which were among the 18 MSAGs or among the 12 most connected intramodular hub genes in module Turquoise.

The first is M190273, encoding an EIN3-like transcription factor. M190273 was assigned to six tight clusters $1,8,9,21,40$ and 45 with $P$. scores 40.7-81.0 (Fig. 8a, Additional file 8: Table S7, Additional file 9: Figure S2). Notably, Lemon-Tree clustered Ma1 into Cluster 1, to which M190273 was assigned as a regulator with the highest $P$. score (81.0) (Fig. 8a), suggesting M190273 might potential regulate Ma1. M190273 was one of the 18 MSAGs (Fig. 5a, Additional file 6: Table S5) and of high MM value (0.866) in module Turquoise. In the six M190273 regulated clusters, the fruit samples were all clustered into two primary clades, which were nearly perfectly alongside the line dividing the two genotypes $\mathrm{Ma}$ and mama (Fig. 8a, Additional file 9: Figure S2). Such clustering pattern of fruit samples were not observed in any of the remainder 15 clusters regulated by the other 11 regulator genes (Fig. 8, Additional files 9, 10: Figures S2, 3), further supporting the potential regulatory role of M190273 in fruit acidity. The second is M525602, encoding a receptor-like protein kinase. M525602 was assigned to cluster 22 with $P$. score 37.8 (Fig. 8b). Similar to M190273, M525602 is also one of the 18 MSAGs (Fig. 5a) that had a high MM value (0.884) in module Turquoise. The third is M319170, one of the 12 intramodular hub genes in module Turquoise (Fig. 6). M319170 was assigned to cluster 0 with $P$. score 36.7 (Fig. 8c). As aforementioned, M319170 encodes an AtIQD2-like $\mathrm{CaM}$ binding protein involved in calcium signaling and behaved similarly as Ma1 in kTotal changes in genotype groups $\mathrm{Ma}$ and mama. 

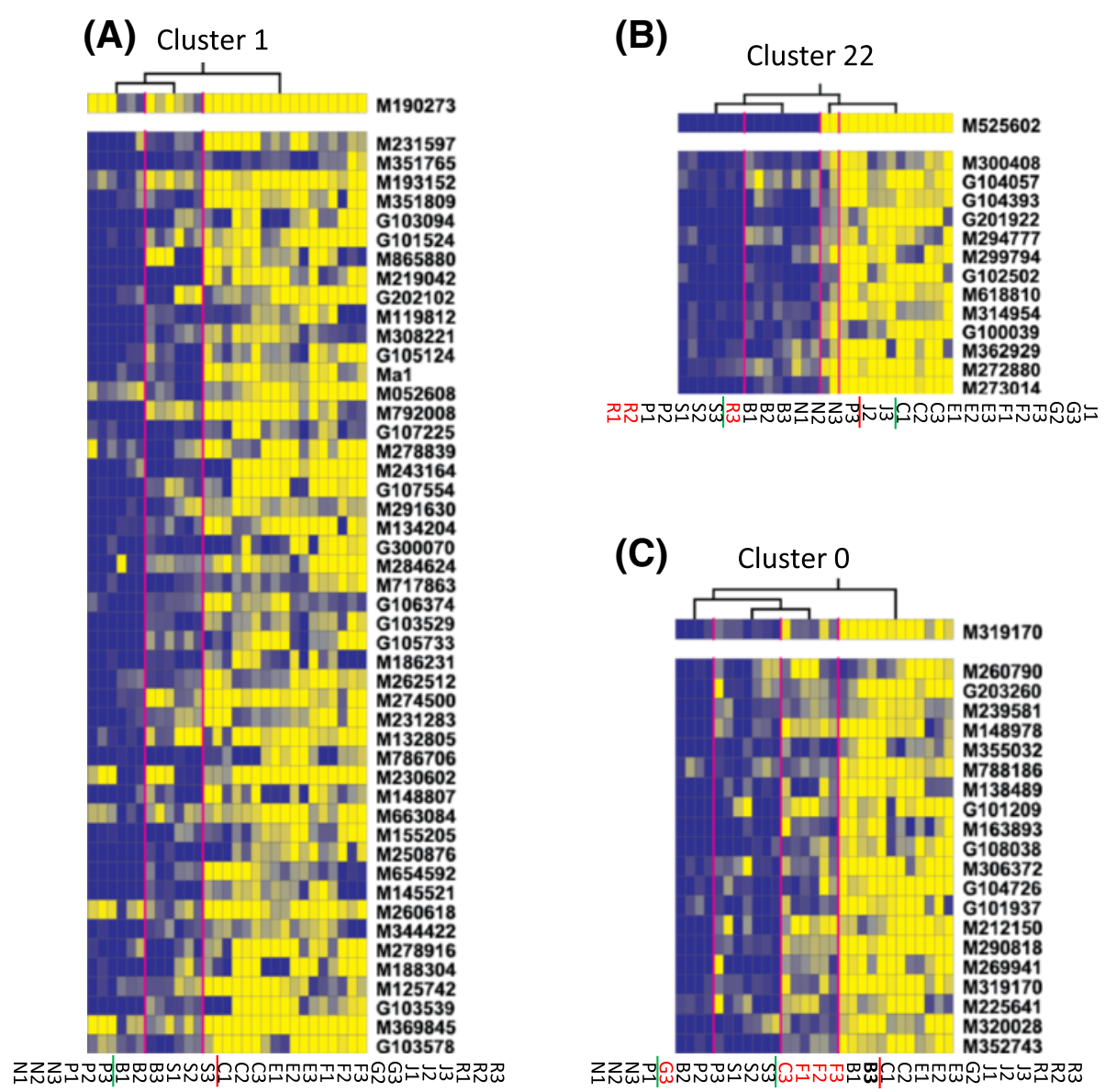

Fig. 8 Regulator genes and their assigned tight clusters. a Regulator M190273 (upper panel) and Cluster 1 (mid panel) of 48 genes. The expression of genes is color coded from low (dark blue) through high (bright yellow). Each row stands for a gene (listed on the right) and each column for a sample named at the bottom (see the legend in Fig. 1 for keys). The regulator is assigned based on the hierarchical tree on top, which indicates how samples were clustered together with the red vertical lines. The short red vertical line in the sample list shows where the two primary clades diverge while the short green vertical line marks where the secondary clades depart within a primary clade. The genotype names in red indicate they are not in agreement with one of the two primary clusters Ma_ or mama. b Regulator M525602 and Cluster 22 of 13 genes. c Regulator M319170 and Cluster 0 of 20 genes. Elements in (b) and (c) and their contents, formats and messages are the same as those in (a)

\section{Detection of enriched GO terms and MapMan (sub-) bins} in network modules

To detect enriched GO terms and MapMan (sub-) bins in the five significant modules, a series of hypergeometric tests were performed against the background of 39,679 expressed genes. Overall, eight GO terms and ten MapMan sub-bins were enriched $\left(\mathrm{P}_{\mathrm{FDR}}<0.05\right)$ in modules Turquoise, Brown, Blue and Yellow, but not in Black, and no terms were repressed (Table 1, Additional file 11: Table S8). Due to multifaceted roles of genes and due to both GO and MapMan systems were used, some of the enriched terms had the same or mostly overlapping member genes. In total, the 18 enriched terms covered 77 non-redundant genes, including 17 in module Turquoise, 9 in Brown, 46 in Blue and 5 in yellow (Table 1, Additional file 11: Table S8).
In Turquoise, two GO (BP) terms (GO:0015979 and GO:0009765) and two MapMan sub-bins (1.3.4 and 1.1.1.1) were enriched, which are all related to photosynthesis (Table 1, Additional file 11: Table S8). However, GO: 0009765 and sub-bin 1.1.1.1 had the same five member genes. Notably, M800352, a member in GO:0015979, is one of the 18 MSAGs (Fig. 5a, Table 1, Additional file 11: Table S8).

In Brown, three GO (BP) terms and one MapMan subbin 10.5.3 (cell wall leucine-rich repeat family protein) were enriched (Table 1, Additional file 11: Table S8). The three GO terms were highly related as the four member genes in GO:0051258 (protein polymerization) and GO:0007017 (microtubule-based process) were identical and were also among the six member genes in GO:0007018 (microtubule-based movement). M192830, a common member 
Table 1 GO terms and/or MapMan (sub-) bines significantly enriched in WGCNA modules significantly correlated with fruit acidity ${ }^{a}$

\begin{tabular}{|c|c|c|c|c|c|c|c|c|}
\hline $\begin{array}{l}\text { WGCNA } \\
\text { Module }\end{array}$ & $\begin{array}{l}\mathrm{GO} / \\
\text { MapMan }\end{array}$ & Code & Description & $\begin{array}{l}\text { No. of } \\
\text { member } \\
\text { genes }\end{array}$ & $P_{F D R}$ & $\begin{array}{l}\text { Important } \\
\text { member gene }\end{array}$ & $\begin{array}{l}\text { Related GO } \\
\text { /MapMan }\end{array}$ & $\begin{array}{l}\text { Enrichment in } \\
\text { developing fruit }^{\mathrm{b}}\end{array}$ \\
\hline \multirow[t]{4}{*}{ Turquoise } & GO (BP) & 0015979 & photosynthesis & 9 & $9.89 \mathrm{E}-03$ & M800352 & $1.1 .1 .2 ; 1.1 .2 .2$ & Yes \\
\hline & GO (BP) & 0009765 & photosynthesis, light harvesting & 5 & $1.19 \mathrm{E}-02$ & & 1.1.1.1 & Yes \\
\hline & MapMan & 1.3.4 & PS.calvin cycle.GAP & 3 & $2.36 \mathrm{E}-02$ & & & Yes \\
\hline & MapMan & 1.1.1.1 & PS.lightreaction.photosystem II.LHC-II & 5 & $3.41 \mathrm{E}-02$ & & 0009765 & Yes \\
\hline \multirow[t]{4}{*}{ Brown } & GO (BP) & 0007018 & microtubule-based movement & 6 & $1.09 \mathrm{E}-03$ & M192830 & $\begin{array}{l}31.1 ; 0051258 ; \\
0007017\end{array}$ & $\begin{array}{l}\text { Yes (by sub-bin } \\
31.1 \text { ) }\end{array}$ \\
\hline & GO (BP) & 0051258 & protein polymerization & 4 & 2.44E-02 & M192830 & $\begin{array}{l}\text { 31.1; } 0007017 ; \\
0007018\end{array}$ & $\begin{array}{l}\text { Yes (by sub-bin } \\
31.1 \text { ) }\end{array}$ \\
\hline & $\mathrm{GO}(\mathrm{BP})$ & 0007017 & microtubule-based process & 4 & $1.93 \mathrm{E}-02$ & M192830 & $\begin{array}{l}31.1 ; 0051258 \\
0007018\end{array}$ & $\begin{array}{l}\text { Yes (by sub-bin } \\
31.1 \text { ) }\end{array}$ \\
\hline & MapMan & 10.5 .3 & cell wall.cell wall proteins.LRR & 3 & $2.96 \mathrm{E}-02$ & & & No \\
\hline \multirow[t]{8}{*}{ Blue } & GO (BP) & 0009607 & response to biotic stimulus & 10 & $2.23 \mathrm{E}-10$ & & 20.2 .99 & Yes ( by 20.2.99) \\
\hline & $\mathrm{GO}$ (MF) & 0016491 & oxidoreductase activity & 19 & 8.27E-03 & $\begin{array}{l}\text { M216638; } \\
\text { M217451 }\end{array}$ & $\begin{array}{l}\text { 16.1.4.2; } \\
0004097\end{array}$ & Yes \\
\hline & GO (MF) & 0004097 & catechol oxidase activity & 3 & $9.94 \mathrm{E}-03$ & & 0016491 & Yes (by 0016491) \\
\hline & MapMan & 20.2.99 & stress.abiotic.unspecified & 11 & 3.33E-08 & & 0009607 & Yes, \\
\hline & MapMan & 20.1 & stress.biotic & 11 & 2.84E-03 & $\begin{array}{l}\text { M782085; } \\
\text { M916930 }\end{array}$ & & No \\
\hline & MapMan & 17.7 .3 & $\begin{array}{l}\text { hormone metabolism.jasmonate. } \\
\text { induced-regulated-responsive- } \\
\text { activated }\end{array}$ & 2 & $9.20 \mathrm{E}-03$ & & & No \\
\hline & MapMan & 11.4 & lipid metabolism.TAG synthesis & 3 & $7.14 \mathrm{E}-03$ & & & No \\
\hline & MapMan & 16.1.4.2 & $\begin{array}{l}\text { secondary } \\
\text { metabolism.isoprenoids.carotenoids. } \\
\text { phytoene dehydrogenase }\end{array}$ & 2 & $1.65 \mathrm{E}-02$ & & 0016491 & Yes \\
\hline \multirow[t]{2}{*}{ Yellow } & MapMan & 11.6 & $\begin{array}{l}\text { lipid metabolism.lipid transfer } \\
\text { proteins etc }\end{array}$ & 3 & $1.91 \mathrm{E}-02$ & M274205 & & No \\
\hline & MapMan & 16.1.3.1 & $\begin{array}{l}\text { secondary metabolism.isoprenoids. } \\
\text { tocopherol biosynthesis. } \\
\text { hydroxyphenylpyruvate dioxygenase }\end{array}$ & 2 & $3.88 \mathrm{E}-02$ & & & No \\
\hline
\end{tabular}

${ }^{a}$ The GO terms and/or MapMan (sub-) bins that have the same or mostly overlapping member genes in the same module are highlighted with same color(s) ${ }^{\mathrm{b}}$ Bai et al. [24]

gene in these GO terms, is one of the 12 hub genes in Brown (Fig. 6).

In Blue, we detected three GO terms and five MapMan sub-bins, the most in this study (Table 1, Additional file 11: Table S8). But the ten member genes in GO:0009607 (response to biotic stimulus) were fully covered by the 11 genes in sub-bin 20.2.99 (abiotic stress unspecified). Similarly, the two genes in sub-bin 16.1.4.2 (carotenoid phytoene dehydrogenase) and three genes in GO:0004097 (catechol oxidase activity) were covered by the 19 genes in GO: 0016491 (oxidoreductase activity). Sub-bins 11.4 (triacylglycerol synthesis), 17.7 .3 (jasmonate responsive) and 20.1 (biotic stress) were unique. Remarkably, four of the 12 hub genes in Blue were present, i.e. M216638 and M217451 in GO: 0016491, and M782085 and M916930 in sub-bin 20.1 (Fig. 6, Table 1, Additional file 11: Table S8).

In Yellow, two MapMan sub-bins 11.6 (lipid transfer proteins) and 16.1.3.1 (hydroxyphenylpyruvate dioxygenase) were enriched, and M274205 from sub-bin 11.6 is one of the 12 hub genes in Yellow (Fig. 6, Table 1, Additional file 11: Table S8).

\section{qRT-PCR confirmation of gene expression}

To evaluate if and how the RPKM values reflected the gene expression levels, a set of eight genes were analyzed using qRT-PCR (Fig. 9). The eight genes include Ma1, the EIN3-like regulator M190273, another most significant gene for acidity M651862 encoding a protein serine/threonine kinase, and five others. The data not only confirmed that the relative expressions of the eight genes in qRT-PCR were significantly $(p=6.663 \mathrm{E}-3$ to 9.647E-5) correlated with their RPKM values in RNAseq, but also confirmed their differential expression between the two genotypes groups $M a_{-}$and mama.

\section{Discussion}

\section{Identification of individual fruit acidity associated genes}

In this study, ten apple varieties were carefully chosen to represent the two genotype groups $M a_{-}$(MaMa and Mama) and mama of contrast fruit acidity levels (normal/ high vs low). These varieties were grafted on the same rootstock P22 and grown in the same orchard with the same management routines to curtail the environmental impacts on gene expression. However, since the apples are of diverse genetic background and matured at varying dates, fruit transcriptome at maturity were inevitably influenced by these genetic and environmental variables. To minimize such biases, three replicates per variety and at least four varieties per genotype group were sampled and used for RNA-seq analysis. This strategy appeared to 


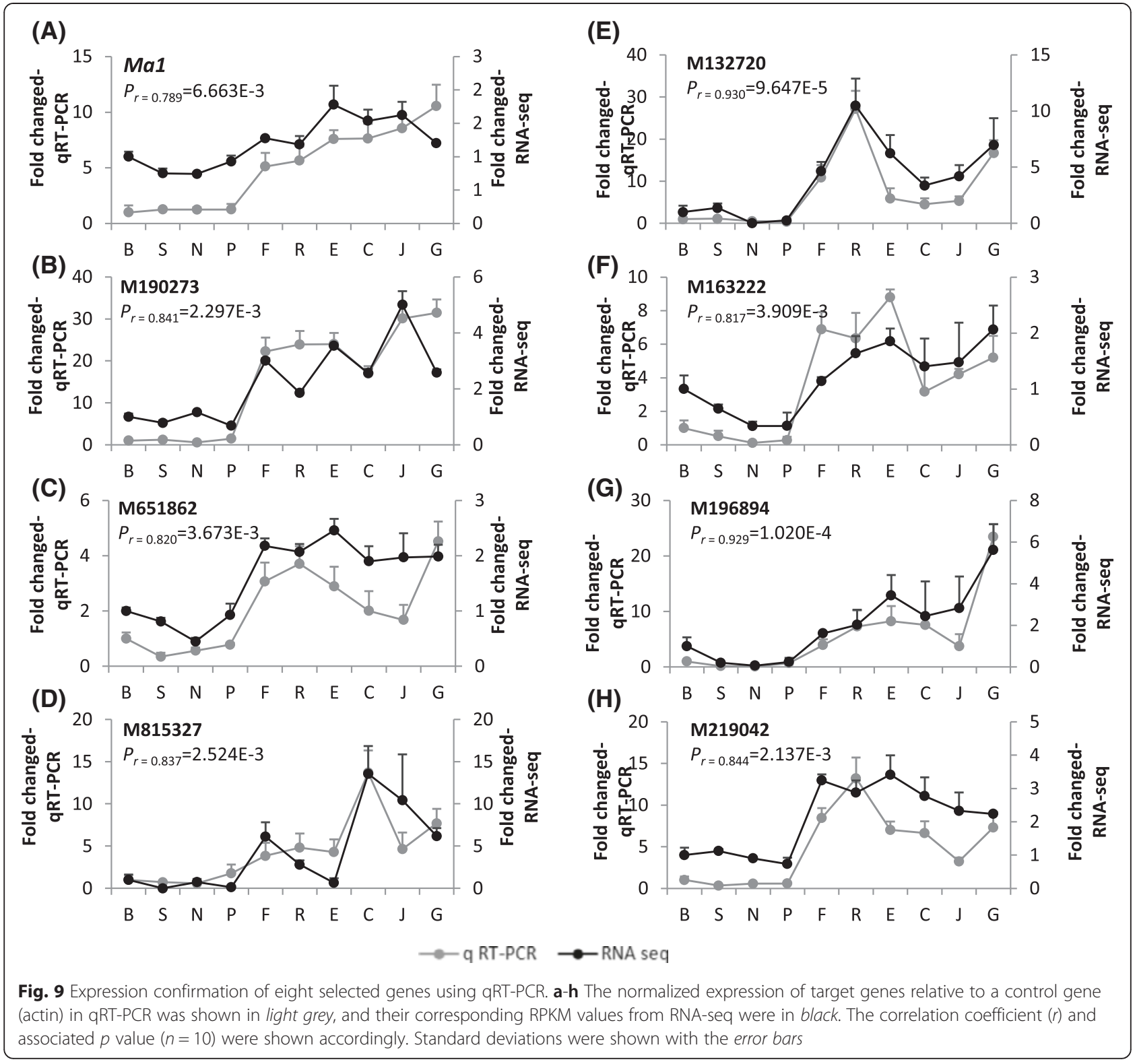

be appropriate and adequate for the defined objectives in this study as we identified 1301 fruit acidity associated genes (FAAGs). In developing fruit of 'Golden Delicious (GD)' of varying malate levels, 3066 differentially expressed genes (DEGs) were identified [24]. In comparison of the two lists of genes, 1050 (80.7 \%) of the 1301 FAAGs were not present in the 3066 DEGs, suggesting most genes relevant for fruit acidity variation across diverse genetic background were largely different from those important for malate variations in the developing fruit of GD. Overall, we found 37,484 genes expressed (RPKM >0.3) in the $M a_{-}$group and 37,813 genes in the mama group. As there were 35,618 genes expressed in both groups, the total number of expressed genes was 39,679 (Fig. 2a). Varying RPKM thresholds were employed in published studies [43, 44], we view RPKM >0.3 used previously [30] fit this set of RNA-seq data well. Of the 39,679 expressed genes, 1301 were regarded FAAGs. Given the significant correlation between RPKM in RNA-seq and relative expression in qRT-PCR in the eight genes tested, we consider that the majority, if not all, of the genes relevant for fruit acidity variation at maturity in this study were included in the 1301 genes.

\section{Co-expression gene network modules and GO terms and} MapMan (sub-) bins relevant for fruit acidity

WGCNA [33, 45] is one of the programs used widely for inferring co-expression network modules [46-49]. Using WGCNA, we identified five modules highly associated with fruit acidity (Fig. 3c). Hypergeometric tests identified eight 
GO terms and ten MapMan (sub-) bins that were enriched in four of the five modules (Table 1, Additional file 11: Table S8).

In the most significant module Turquoise that includes Ma1, the two enriched GO terms and two MapMan sub-bins are all related to photosynthesis (Table 1, Additional file 11: Table S8). In the developing fruit, the two GO terms and the two MapMan sub-bins were also enriched [24], and 11 (64.7\%) of the 17 member genes in the four enriched terms were identified in both studies (Table 1, Additional file 11: Table S8). This suggested that fruit photosynthesis could likely be a part of the mechanism for the contrast malate levels between the two genotype groups at maturity. It was proposed that the relative more active photosynthesis in young fruit may facilitate malate biosynthesis, thereby contributing to higher malate levels in young fruit than in maturing fruit [24]. However, chloroplasts are only present in the hypodermal green tissues and the inner perivascular tissues in mature apple fruit, and are essentially absent in parenchyma cells that constitute the major portion of flesh [50, 51]. How enhanced photosynthesis activities in a fraction of cells would lead to increased overall malate levels in fruit remains to be understood.

In module Brown, the enrichment of MapMan sub-bin 10.5.3 (cell wall leucine-rich repeat family protein) was unique to this study. However, the three enriched GO terms (GO:0007018, GO:0051258 and GO:0007017) were essentially a reflection of MapMan sub-bin 31.1 (cell organization) (Table 1, Additional file 11: Table S8), which was enriched in negative malate dependent manner in developing fruit [24]. In module Blue, the enrichment of MapMan sub-bins 11.4 (triacylglycerol synthesis), 17.7.3 (jasmonate responsive) and 20.1 (biotic stress) were identified in this study only. But GO:0016491 (oxidoreductase activity), and MapMan sub-bins 20.2.99 (abiotic stress unspecified) and 16.1.4.2 (carotenoid phytoene dehydrogenases) were also enriched in developing fruit [24]. In module Yellow, the enrichment of two MapMan sub-bins 11.6 (lipid transfer proteins) and 16.1.3.1 (hydroxyphenylpyruvate dioxygenases) was unique in these diverse mature fruit.

Overall, 12 enriched terms (covering 53 of the 77 genes), including four from Turquoise (17 genes), three from Brown (6 genes), and five from Blue (30 genes), were also enriched in developing fruit of 'Golden Delicious' [24], whereas the remainder six terms (covering 24 of the 77 genes) were enriched specifically in this study (Table 1 , Additional file 11: Table S8). This suggested that common and unique processes relevant for malate variations in both the diverse mature fruit and the developing fruit of 'Golden Delicious' exist. However, 27 of the 30 genes in Blue and all of the six genes in Brown were not found to be relevant in developing fruit although 11 of the 17 genes related to photosynthesis in Turquoise were (Table 1, Additional file 11: Table S8), suggesting the common processes may operate but most likely with their unique gene sets.

\section{Transcriptional regulation of fruit acidity mediated by Ma1}

In an ongoing survey of the USDA Malus repository at Geneva, New York, we identified 40 diverse apple accessions of genotype ma1ma1 using marker CAPS $_{1455}$ that can detect the stop codon leading SNP in ma1 [10] and found that each of them had fruit $\mathrm{pH}>4.0$ (Xu, unpublished data), a typical low fruit acidity characteristic in genotype group mama. These data, together with the previous studies $[4,10,11]$, suggested us that Ma1, a member of the ALMT1 gene family [52], is the genetic determinant underlying the $\mathrm{Ma}$ locus controlling acidity levels in mature apple fruit although the functional genetic complementation data are not available.

Ma1 is thought to exert its effect on fruit acidity by both the truncation leading mutation and expression levels, where the mutation plays greater role [10]. In this study, Ma1 showed not only high gene significance for malate (Fig. 5a), but also high intramodular connectivity in module Turquoise (Figs. 6, 7), further supporting this notion. However, in our previous effort to understand the developmental regulation of malate levels in the developing fruit of 'Golden Delicious', Ma1 was not present in the major co-expression gene network due to its less significant $(p=0.03)$ correlation with malate although the involvement of Ma1 was clear [24]. This is likely caused by the fact that the study focused on developing fruit of a single variety ('Golden Delicious') of genotype Mama that had malate levels equivalent to the normal to high acidity range during fruit development, i.e. the truncation mutation effect of $M a 1$ for low acidity in genotype group mama was absent.

The current models accounting for the ALMT1 mediated plant tolerance to soil aluminum toxicity in Arabidopsis and rice comprise several common elements [53, 54]. These elements include environmental stimuli $\left(\mathrm{Al}^{3+} / \mathrm{H}^{+}\right)$, a receptor (unknown) on the plasma membrane that interacts with $\mathrm{Al}^{3+} / \mathrm{H}^{+}$to initiate a signal transduction pathway (unknown), transcription factors (STOP1 in Arabidopsis and ART1 in rice, both of which are $\mathrm{C}_{2} \mathrm{H}_{2}$ zinc finger transcription factors, i.e. $\mathrm{TFs}$ ), protein kinase (unknown) activating the TFs by phosphorylation, and responsive genes ALMT1, ABC transporters (ASL1, ASL3, STAR1) and others [53, 54]. In the developing fruit of GD, several groups of genes functionally similar to these elements were co-enriched or co-suppressed in a malate dependent manner, including $14 \mathrm{C}_{2} \mathrm{H}_{2}$ transcriptional factors, 27 protein kinases, and 23 receptor kinases for signaling [24]. In addition, G105811, one of $14 \mathrm{C}_{2} \mathrm{H}_{2}$ transcription factors, was annotated as a 
STOP1-like protein by Mercator [40]. These had led to a speculation that a similar transcriptional regulation model for malate in developing fruit may exist although ALMT1 targets plasma membrane [24].

In this study, some of these functionally similar components were also identified. For example, within the 18 MSAGs, we see M525602 encoding a leucine-rich repeat (LLR) transmembrane receptor protein kinase for signaling, M651862 a protein serine/threonine kinase, M282275 an ABC transporter, and of cause Ma1 an ALMT1 like malate transporter (Fig. 5a). Interestingly, Lemon-Tree based regulator analysis identified the LLR receptor kinase encoding gene M525602 as a regulator of Cluster 22 of 13 genes (Fig. 8b). Moreover, M250124, the most intramodular connected gene in module Turquoise (Fig. 6), encodes a protein kinase. These data suggested that the ALMT1 mediated plant tolerance models somewhat be plausible for the transcriptional regulation of fruit acidity. However, this study identified only one $\mathrm{C}_{2} \mathrm{H}_{2}$ zinc finger TF (M302279) in the 1301 FAAGs assigned to module Red not highly correlated with acidity. There is no evidence for $\mathrm{C}_{2} \mathrm{H}_{2}$ zinc finger TFs being part of the transcriptional regulation of Ma1 mediated fruit acidity.

Identification of regulator M190273 by Lemon-Tree may have suggested an emerging mechanism for fruit acidity regulatory network mediated by Ma1. Again, M190273 is one of the MSAGs (Fig. 5a) as well as a highly connected gene in module Turquoise (Fig. 7). Lemon-Tree assigned M190273 as a regulator to six clusters of 183 genes in total, including Cluster 1 containing Ma1 (Fig. 8a), Cluster 40 containing G104764 and M345601 (Additional file 9: Figure S2C), and Cluster 21 containing M651862 (Additional file 9: Figure S2D), which are all among the 18 MSAGs (Fig. 5a). Although G104764 and M345601 are of unknown function, M651862 was speculated as a possible protein kinase component in the ALMT1 model aforementioned. Another relevant observation is that samples in these six clusters were all clustered alongside the line between the two genotype groups $\mathrm{Ma}$ and mama, a pattern not observed for any other regulators, suggesting M190273 is likely a critical regulator for fruit acidity. M190273 shows some sequence similarity to EIN3-like genes, which are transcription factors important in ethylene response and signal transduction in plant $[55,56]$. Previous studies documented that the root growth inhibition by aluminum stress is mediated by ethylene in bean [57], Lotus japonicus [58], and Arabidopsis is [59]. A more recent study reported that aluminuminduced malate efflux from wheat roots and tobacco cells transformed with TaALMT1 was negatively regulated by ethylene although the molecular mechanisms remain unknown [60]. These reports hint that it is possible for M190273 to be a regulator of malate in apple fruit.
M319170, one of the 12 hub genes in module Turquoise (Fig. 6), is a regulator assigned to Cluster 0 containing M225641, one of the 18 MSAGs (Fig. 5a). M319170 encodes a CaM binding protein similar to AtIQD2 involved in calcium $\left(\mathrm{Ca}^{2+}\right)$ signaling [61]. M140330, another hub gene of module Turquoise (Fig. 6), encodes a protein similar to CaM-like3 (CML3) of role in calcium signaling [62]. Among the 18 MSAGs (Fig. 5a), M834327 encodes a calcium regulated channel similar to AtCNGC 20. It has been demonstrated that AtCNGC20 is localized to tonoplast [63] as well as plasma membrane [64], and to interact with all $\mathrm{CaM}$ isoforms through its IQ domain [64]. In a study investigating carbohydrate metabolism in two apple genotypes that sharply differ in malate accumulation in a way similar to those between the two genotype groups $\mathrm{Ma}$ and mama found that calcium contents were significant higher in high acid fruit than in low acid fruit [65]. These observations suggested $\mathrm{Ca}^{2+}$ signaling might also be an important direction for better understanding the regulation of malate levels in mature apple fruit. Interestingly, $\mathrm{Ca}^{2+}$ signals have also been considered one of the possible signal transduction pathways for aluminum tolerance lately [54].

\section{Conclusions}

The most relevant finding of this study is the identification of a weighted gene co-expression network analysis (WGCNA) module (Turquoise) of 336 genes that not only encompasses Ma1 but also shows the highest modular correlation with acidity in mature fruit of diverse genetic background. Based on LemonTree algorisms, MDP0000190273, which putatively encodes an EIN3-like transcriptional factor, is likely the most important regulator of $M a 1$ and its mediated gene network. The two gene ontology biological process terms (GO:0015979 and GO:0009765) and two MapMap sub-bins (1.3.4 and 1.1.1.1) that were significantly enriched in module Turquoise implicated that photosynthesis related pathways are likely essential for acidity. Overall this study for the first time provides not only important insight into the Ma1-mediated gene network controlling acidity in mature apple fruit of diverse genetic background, but also relevant clues for future biological validation, including the three putative regulators, MDP0000190273, MDP0000319170 and MDP0000140330.

\section{Supporting data}

The data sets supporting the results of this article are available in the NCBI Sequence Read Archive (SRA) repository (http://www.ncbi.nlm.nih.gov/Traces/sra/) un der accession SRX673837. 


\section{Additional files}

Additional file 1: Table S1. Fruit harvest date and weight.

Additional file 2: Table S2. Overview of RNA-seq reads mapping. (DOCX $31 \mathrm{~kb}$ )

Additional file 3: Table S3. List of primers used in qRT-PCR. (DOCX $29 \mathrm{~kb}$ )

Additional file 4: Table S4. List of the 1301 FAAGs and their RPKM values and assignment in WGCNA modules. (XLSX 550 kb)

Additional file 5: Figure S1. Analysis of modules Black, Brown, Blue and Yellow. (A) Module eigengene values across the 29 samples, including 17 in $\mathrm{Ma}_{-}$on left and 12 in mama on right. Samples are represented by the combination of a letter (abbreviated cultivar name) and a number (replicate) (see legends in Fig. 1, 4 for keys). (B) Correlation between module membership (MM) and gene significance (GS) for malate. (PPTX $75 \mathrm{~kb})$

Additional file 6: Table S5. List of the most significant acidity genes (MSAGs). (DOCX $31 \mathrm{~kb}$ )

Additional file 7: Table S6. List of intramodular hub genes in modules highly associated with acidity. (XLSX $26 \mathrm{~kb}$ )

Additional file 8: Table S7. Regulator genes identified by Lemon-Tree. (DOCX $31 \mathrm{~kb}$ )

Additional file 9: Figure S2. Regulator M190273 and its other five assigned tight clusters. Elements and their contents, formats and messages are same as those noted in Fig. 8a. (A) Cluster 8 of 31 genes. (B) Cluster 9 of 28 genes. (C) Cluster 40 of 55 genes. (D) Cluster 21 of 11 genes. (E) Cluster 45 of 10 genes. (PPTX $238 \mathrm{~kb}$ )

Additional file 10: Figure S3. Other regulators from modules Turquoise and Brown and their assigned tight clusters. Elements and their contents, formats and messages are same as those noted in Fig. 8a. (A) Regulator M239684 and Cluster 41 of 68 genes. (B) Regulator M239684 and Cluster 5 of 14 genes. (C) Regulator M239684 and Cluster 7 of 14 genes. (D) Regulator M753318 and Cluster 23 of 11 genes. (E) Regulator M753318 and Cluster 32 of 11 genes. (F) Regulator M175481 and Cluster 2 of 16 genes. (G) Regulator M134341 and Cluster 42 of 12 genes. (PPTX $213 \mathrm{~kb}$ )

Additional file 11: Table S8. Enriched gene ontology (GO) terms and MapMan (sub)-bins in WGCNA modules. (XLSX $16 \mathrm{~kb}$ )

\section{Abbreviations}

ALMT1: Aluminum-activated Malate Transporter1; DEGs: Differentially expressed genes; FAAGs: Fruit acidity associated genes; FDR: False discovery rate; GD: Golden Delicious; Ma1: Malic acid1; MSAGs: Most significant acidity genes; QTL: Quantities trait locus; RNA-seq: mRNA sequencing; RPKM: Reads per kilobase of exon model per million mapped reads; TA: Titratable acidity.

\section{Competing interests}

The authors declare that they have no competing interests.

\section{Authors' contributions}

YB and LD carried out fruit sampling, acidity and metabolite evaluation, RNA isolation and RNA-seq library construction and qPCR validation of RNA-Seq. LC and GZ participated in the design and coordination of the study. KX conceived the study. YB and KX analyzed the data and wrote the manuscript. All authors read and approved the final manuscript.

\section{Acknowledgements}

We thank Dr. Herb Aldwinckle for providing the fruit samples used in this study and Dr. Zhangjun Fei for helpful discussions in RNA-seq data analyses. This work was supported in part by the Agriculture and Food Research Initiative competitive grant no. 2014-67013-21660 of the USDA National Institute of Food and Agriculture (NIFA).

\section{Author details}

${ }^{1}$ Horticulture Section, School of Integrative Plant Science, Cornell University, New York State Agricultural Experiment Station, Geneva, NY 14456, USA.
${ }^{2}$ Horticulture Section, School of Integrative Plant Science, Cornell University, Ithaca, NY 14853, USA. ${ }^{3}$ USDA-ARS, Plant Genetic resource and Grape Genetic Research Units, Geneva, NY 14456, USA.

Received: 21 March 2015 Accepted: 5 August 2015

Published online: 16 August 2015

\section{References}

1. Zhang YZ, Li PM, Cheng LL. Developmental changes of carbohydrates, organic acids, amino acids, and phenolic compounds in 'Honeycrisp' apple flesh. Food Chem. 2010;123(4):1013-8.

2. Yamaki S. Isolation of vacuoles from immature apple fruit flesh and compartmentation of sugars, organic acids, phenolic compounds and amino acids. Plant Cell Physiol. 1984;25(1):151-66.

3. Hulme AC, Wooltorton LSC. The organic acid metabolism of apple fruits: changes in individual acids during growth on the tree. J Sci Food Agr. 1957:8:117-22

4. Xu K, Wang A, Brown S. Genetic characterization of the Ma locus with $\mathrm{pH}$ and titratable acidity in apple. Mol Breeding. 2012;30(2):899-912.

5. Maliepaard C, Alston FH, van Arkel G, Brown LM, Chevreau E, Dunemann F, et al. Aligning male and female linkage maps of apple (Malus pumila Mill) using multi-allelic markers. Theor Appl Genet. 1998;97(1-2):60-73.

6. Kenis K, Keulemans J, Davey M. Identification and stability of QTLs for fruit quality traits in apple. Tree Genet Genomes. 2008;4(4):647-61.

7. Liebhard R, Kellerhals M, Pfammatter W, Jertmini M, Gessler C. Mapping quantitative physiological traits in apple (Malus $\mathrm{x}$ domestica Borkh). Plant Mol Biol. 2003;52(3):511-26.

8. Zhang Q, Ma B, Li H, Chang Y, Han Y, Li J, et al. Identification, characterization, and utilization of genome-wide simple sequence repeats to identify a QTL for acidity in apple. BMC Genomics. 2012;13(1):537.

9. Kumar S, Chagne D, Bink MCAM, Volz RK, Whitworth C, Carlisle C. Genomic selection for fruit quality traits in apple (Malus $\mathrm{x}$ domestica Borkh). PLoS One. 2012;7(5):e36674.

10. Bai Y, Dougherty L, Li M, Fazio G, Cheng L, Xu K. A natural mutation-led truncation in one of the two aluminum-activated malate transporter-like genes at the Ma locus is associated with low fruit acidity in apple. Mol Genet Genomics. 2012;287(8):663-78.

11. Khan S, Beekwilder J, Schaart J, Mumm R, Soriano J, Jacobsen E, et al. Differences in acidity of apples are probably mainly caused by a malic acid transporter gene on LG16. Tree Genet Genomes. 2013;9:475-87.

12. Mortazavi A, Williams BA, McCue K, Schaeffer L, Wold B. Mapping and quantifying mammalian transcriptomes by RNA-Seq. Nat Methods. 2008;5(7):621-8.

13. Wilhelm BT, Landry JR. RNA-Seq-quantitative measurement of expression through massively parallel RNA-sequencing. Methods. 2009;48(3):249-57.

14. Lister R, O'Malley RC, Tonti-Filippini J, Gregory BD, Berry CC, Millar AH, et al. Highly integrated single-base resolution maps of the epigenome in Arabidopsis. Cell. 2008;133(3):523-36.

15. Zenoni S, Ferrarini A, Giacomelli E, Xumerle L, Fasoli M, Malerba G, et al. Characterization of transcriptional complexity during berry development in Vitis vinifera using RNA-Seq. Plant Physiol. 2010;152(4):1787-95.

16. Li P, Ponnala L, Gandotra N, Wang L, Si Y, Tausta SL, et al. The developmental dynamics of the maize leaf transcriptome. Nat Genet. 2010;42(12):1060-7.

17. Zhang G, Guo G, Hu X, Zhang Y, Li Q, Li R, et al. Deep RNA sequencing at single base-pair resolution reveals high complexity of the rice transcriptome. Genome Res. 2010;20(5):646-54.

18. Krost C, Petersen R, Lokan S, Brauksiepe B, Braun P, Schmidt E. Evaluation of the hormonal state of columnar apple trees (Malus $\mathrm{x}$ domestica) based on high throughput gene expression studies. Plant Mol Biol. 2013;81(3):211-20.

19. Krost C, Petersen R, Schmidt ER. The transcriptomes of columnar and standard type apple trees (Malus x domestica)-a comparative study. Gene. 2012;498(2):223-30.

20. Zhang Y, Zhu J, Dai H. Characterization of transcriptional differences between columnar and standard apple trees using RNA-Seq. Plant Mol Biol Report. 2012;30(4):957-65.

21. Gapper NE, Rudell DR, Giovannoni JJ, Watkins CB. Biomarker development for external $\mathrm{CO}_{2}$ injury prediction in apples through exploration of both transcriptome and DNA methylation changes. AoB Plants. 2013;5.

22. Xia R, Zhu H, An Y-Q, Beers E, Liu Z. Apple miRNAs and tasiRNAs with novel regulatory networks. Genome Biol. 2012;13(6):R47. 
23. Otto D, Petersen R, Brauksiepe B, Braun P, Schmidt E. The columnar mutation ("Co gene") of apple (Malus $\times$ domestica) is associated with an integration of a Gypsy-like retrotransposon. Mol Breeding. 2014;33:863-80.

24. Bai Y, Dougherty L, Cheng L, Xu K. A co-expression gene network associated with developmental regulation of apple fruit acidity. Mol Genet Genomics. 2015;290(4):1247-63.

25. Bai $Y$, Dougherty L, Xu K. Towards an improved apple reference transcriptome using RNA-seq. Mol Genet Genomics. 2014;289(3):427-38.

26. Jung S, Ficklin SP, Lee T, Cheng C-H, Blenda A, Zheng P, et al. The Genome Database for Rosaceae (GDR): year 10 update. Nucleic Acids Res. 2014;42(D1):D1237-44.

27. Blanpied GD, Silsby KJ. Predicting harvest date windows for apples. In: Information bulletin 221. Ithaca, NY, USA: Cornell Cooperative Extension, Cornell University; 1992.

28. Langmead B, Trapnell C, Pop M, Salzberg SL. Ultrafast and memory-efficient alignment of short DNA sequences to the human genome. Genome Biol. 2009:10(3):R25

29. Quast C, Pruesse E, Yilmaz P, Gerken J, Schweer T, Yarza P, et al. The SILVA ribosomal RNA gene database project: improved data processing and web-based tools. Nucleic Acids Res. 2013;41(Database issue):D590-6.

30. Kang C, Darwish O, Geretz A, Shahan R, Alkharouf N, Liu Z. Genome-scale transcriptomic insights into early-stage fruit development in woodland strawberry Fragaria vesca. Plant Cell. 2013;25(6):1960-78.

31. Baggerly KA, Deng L, Morris JS, Aldaz CM. Differential expression in SAGE: accounting for normal between-library variation. Bioinformatics. 2003;19(12):1477-83

32. Benjamini Y, Hochberg Y. Controlling the false discovery rate-a practical and powerful approach to multiple testing. J R Stat Soc Ser B-Methodol. 1995;57(1):289-300.

33. Langfelder P, Horvath S. WGCNA: an R package for weighted correlation network analysis. BMC Bioinformatics. 2008;9(1):559.

34. Saito R, Smoot ME, Ono K, Ruscheinski J, Wang P-L, Lotia S, et al. A travel guide to Cytoscape plugins. Nat Meth. 2012;9(11):1069-76.

35. Assenov Y, Ramírez F, Schelhorn S-E, Lengauer T, Albrecht M. Computing topological parameters of biological networks. Bioinformatics. 2008;24(2):282-4.

36. Bonnet E, Calzone L, Michoel T. Integrative multi-omics module network inference with Lemon-Tree. arXiv preprint. 2014;arXiv:14080472.

37. Joshi A, De Smet R, Marchal K, Van de Peer Y, Michoel T. Module networks revisited: computational assessment and prioritization of model predictions. Bioinformatics. 2009;25(4):490-6.

38. Joshi A, Van de Peer Y, Michoel T. Analysis of a Gibbs sampler method for model-based clustering of gene expression data. Bioinformatics. 2008:24(2):176-83.

39. Thimm O, Blasing O, Gibon Y, Nagel A, Meyer S, Kruger P, et al. MAPMAN: a user-driven tool to display genomics data sets onto diagrams of metabolic pathways and other biological processes. Plant J. 2004;37(6):914-39.

40. Lohse M, Nagel A, Herter T, May P, Schroda M, Zrenner R, et al. Mercator: a fast and simple web server for genome scale functional annotation of plant sequence data. Plant Cell Environ. 2014;37:1250-8.

41. Götz S, García-Gómez JM, Terol J, Williams TD, Nagaraj SH, Nueda MJ, et al. High-throughput functional annotation and data mining with the Blast2GO suite. Nucleic Acids Res. 2008;36(10):3420-35

42. Falcon S, Gentleman R. Using GOstats to test gene lists for GO term association. Bioinformatics. 2007;23(2):257-8.

43. Kellner R, Bhattacharyya A, Poppe S, Hsu TY, Brem RB, Stukenbrock EH. Expression profiling of the wheat pathogen Zymoseptoria tritici reveals genomic patterns of transcription and host-specific regulatory programs. Genome Biol Evol. 2014;6(6):1353-65.

44. Bagnaresi $P$, Biselli $C$, Orrù L, Urso S, Crispino L, Abbruscato $P$, et al. Comparative transcriptome profiling of the early response to Magnaporthe oryzae in durable resistant vs susceptible rice (Oryza sativa L.) genotypes. PLoS One. 2012;7(12):e51609.

45. Zhang B, Horvath S. A general framework for weighted gene co-expression network analysis. Stat Appl Genet Mol. 2005;4(1):17.

46. Wang YXR, Huang $H$. Review on statistical methods for gene network reconstruction using expression data. J Theor Biol. 2014;362:53-61.

47. Kogelman LJ, Cirera S, Zhernakova DV, Fredholm M, Franke L, Kadarmideen $\mathrm{HN}$. Identification of co-expression gene networks, regulatory genes and pathways for obesity based on adipose tissue RNA sequencing in a porcine model. BMC Med Genomics. 2014;7(1):57.
48. Hollender CA, Kang C, Darwish O, Geretz A, Matthews BF, Slovin J, et al. Floral transcriptomes in woodland strawberry uncover developing receptacle and anther gene networks. Plant Physiol. 2014;165(3):1062-75.

49. Miller JA, Ding S-L, Sunkin SM, Smith KA, Ng L, Szafer A, et al. Transcriptional landscape of the prenatal human brain. Nature. 2014;508(7495):199-206.

50. Phan CT. All-granal chloroplasts of apple-fruit. In: Sybesma C, editor. Advances in photosynthesis research vol. 3. Netherlands: Springer; 1984. p. 63-6.

51. Blanke MM, Lenz F. Fruit photosynthesis. Plant Cell Environ. 1989;12(1):31-46.

52. Barbier-Brygoo H, De Angeli A, Filleur S, Frachisse J-M, Gambale F, Thomine S, et al. Anion channels/transporters in plants: from molecular bases to regulatory networks. Annu Rev Plant Biol. 2011;62(1):25-51.

53. Delhaize E, Ma JF, Ryan PR. Transcriptional regulation of aluminium tolerance genes. Trends Plant Sci. 2012;17:341-8.

54. Liu J, Piñeros MA, Kochian LV. The role of aluminum sensing and signaling in plant aluminum resistance. J Integr Plant Biol. 2014;56(3):221-30.

55. Chao QM, Rothenberg M, Solano R, Roman G, Terzaghi W, Ecker JR. Activation of the ethylene gas response pathway in Arabidopsis by the nuclear protein ETHYLENE-INSENSITIVE3 and related proteins. Cell. 1997;89(7):1133-44.

56. Stepanova AN, Alonso JM. Ethylene signaling and response: where different regulatory modules meet. Curr Opin Plant Biol. 2009;12(5):548-55.

57. Massot N, Nicander B, Barcelo J, Poschenrieder C, Tillberg E. A rapid increase in cytokinin levels and enhanced ethylene evolution precede $\mathrm{Al}^{3+}$-induced inhibition of root growth in bean seedlings (Phaseolus vulgaris L). Plant Growth Regul. 2002;37(2):105-12.

58. Sun P, Tian Q-Y, Zhao M-G, Dai X-Y, Huang J-H, Li L-H, et al. Aluminuminduced ethylene production is associated with inhibition of root elongation in Lotus japonicus L. Plant Cell Physiol. 2007:48(8):1229-35.

59. Sun $\mathrm{P}$, Tian Q-Y, Chen J, Zhang W-H. Aluminium-induced inhibition of root elongation in Arabidopsis is mediated by ethylene and auxin. J Exp Bot. 2010;61(2):347-56

60. Tian Q, Zhang X, Ramesh S, Gilliham M, Tyerman SD, Zhang W-H. Ethylene negatively regulates aluminium-induced malate efflux from wheat roots and tobacco cells transformed with TaALMT1. J Exp Bot. 2014;65(9):2415-26.

61. Abel S, Bürstenbinder K, Müller J. The emerging function of IQD proteins as scaffolds in cellular signaling and trafficking. Plant Signal Behav. 2013;8(6), e24369.

62. McCormack E, Tsai Y-C, Braam J. Handling calcium signaling: Arabidopsis CaMs and CMLs. Trends Plant Sci. 2005;10(8):383-9.

63. Yuen CCY, Christopher DA. The group IV-A cyclic nucleotide-gated channels, CNGC19 and CNGC20, localize to the vacuole membrane in Arabidopsis thaliana. AoB Plants. 2013;5.

64. Fischer C, Kugler A, Hoth S, Dietrich P. An IQ domain mediates the interaction with calmodulin in a plant cyclic nucleotide-gated channel. Plant Cell Physiol. 2013;54(4):573-84

65. Beruter J. Carbohydrate metabolism in two apple genotypes that differ in malate accumulation. J Plant Physiol. 2004;161(9):1011-29.

\section{Submit your next manuscript to BioMed Central and take full advantage of:}

- Convenient online submission

- Thorough peer review

- No space constraints or color figure charges

- Immediate publication on acceptance

- Inclusion in PubMed, CAS, Scopus and Google Scholar

- Research which is freely available for redistribution 